\title{
Research on Survivability Evaluation of Ad Hoc Network Connectivity
}

\author{
WANG Ji-Lu \\ Department of Computer Science and Technology, Beijing Foreign Studies University, Beijing \\ 100089, China \\ wjlgax@sohu.com
}

Keywords: Ad Hoc, Connectivity, Survivability, CTMC, Queuing theory

\begin{abstract}
Connectivity is the basis and premise of the network services. Ad hoc network has the property of topological changes, which makes the connecting reliability the basis of its survivability evaluation. To compute the connecting reliability of Ad hoc network, it needs to analyze the whole system and evaluate the probability of keeping links available. Therefore, by applying the Markov process in Ad hoc network system modeling, the paper analyzes the connection availability of stead-state links when there are system exceptions. When the exception is thrown, it can analyze the phenomenon of instantaneous packet losses and delay at a network node by means of queuing theory. With a combination of steady-state availability analysis and nodes' instantaneous non-response, the paper can draw a conclusion that evaluation indicators like connecting probabilities, packet losses and delay, can be used in survivability evaluation of Ad hoc network.
\end{abstract}

\section{Overview}

Researches into network survivability were mainly about quantitative and qualitative analysis [1]. The document [2] made summary of survivable technology. As for survivability analysis, Dongyan Chen [3] analyzed the performance (stability, delay and failure rate) of Ad hoc network by continuous-time Markov chain; John Koroma [4] built a general modeling of survivability, for example, he built an end-to-end continuous-time Markov chain in Ad hoc network to analyze survivability. The document [5] made an analysis of steady-state availability of Ad hoc network using Markov chain. Through simulation, Krishna Paul [6] studied how parameter like wireless transmission range of nodes, the number of nodes within certain area and the average number of adjacent nodes influence system survivability. The document [7] built a modeling for Ad hoc network by means of random Petri network, and by evaluating connecting probability of nodes under fault conditions and success rate of fixed-length data transmission to analyze the survivability.

Survivability refers to an ability of accomplishing its key assignments promptly while the system is attacked or faults and exceptions are thrown [8]. The document [9] gave a further qualitative description of system survivability. Specifying the definition of network survivability by T1A1.2 [11] made the ability of providing quantitative network services possible, especially under exception conditions like nodes or link failure and being attacked.

What the Ad hoc network focused was on the frequency of exception, the duration of exception and exception's influence on network. The article was written basing on above-mentioned theories and come out with a modeling of Ad hoc network. First, build a model for Ad hoc network on steady-state survivability using continuous-time Markov process. Then, when exceptions occurred, make an instantaneous analysis at nodes using queuing theory. So get the instant packet losses and delay. Finally, combine steady-state availability analysis and nodes' instantaneous analysis into a complete model, which could be used in analysis of system performance like connecting availability, packet losses and delay.

\section{Research on Survivability Evaluation model of Ad Hoc Network Connectivity}

The establishment of connecting availability model. 
Ad hoc network has a property for multiple hop transmission. (shown in Figure 1). In the diagram, every circle represents a cluster. The section in which two clusters join is called intersection region. Assumed that within the same cluster, direct communication among nodes is possible, and data transmission among different clusters has to be forwarded by routing nodes in intersection regions.

Firstly, this article analyses data transmission process in Ad hoc network in terms of double hop transmission. Then, based on reliability theory, it makes further analysis and calculation on data transmission process in terms of multiple hop transmission.

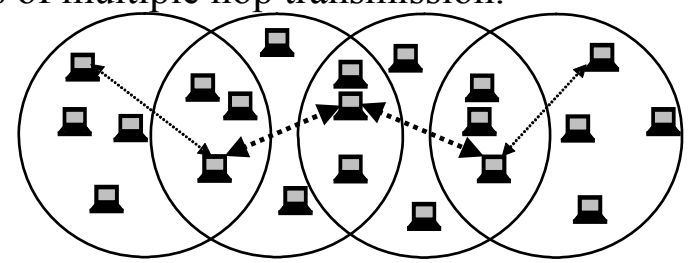

Figure 1 multiple hop transmission in Ad hoc network

Firstly, it should make some assumptions:

- Assumed that fault events are independent from each others, faults could be divided into three main categories: the first kind is node failure, like routing nodes which should be in intersection regions being removed from intersection regions, node transform fault, routing nodes fault and hardware faults; the second type is power failure. For example, due to the limited life of battery, routing nodes are powerless or run down which causes node failure; the third kind is link failures, like links being blocked and failure caused by excessive noise in communication links. There are also other faults, like routing transfer faults, software and hardware faults and managerial faults.

- The occurring times of every type of faults follow exponential distributions.

- Probabilities of fault recovery of different types are the same.

- Transfer delay is less than average time of routing faults. That is no faults during transfer delay.

- Nodes have the same transmission radius.

- Data transmission among different clusters has to be forwarded by routing nodes in intersection regions.

The characteristic of transmission in Ad hoc network is similar to Markov process. Therefore, continuous-time Markov chain could be used to describe the transmission. Furthermore, connection state of links can be also described by Markov chain model. There are several definitions:

Definition 1: Ad hoc system model could be mapped to a finite state machine whose CTMC model state is represented by triad $\{\mathrm{I}, \mathrm{J}, \mathrm{K}\}$.

Definition 2: $\mathrm{i} \in \mathrm{I}=\{0,1,2,3 \ldots \mathrm{N}\}$ represents the number of available routers or available routing nodes $(\mathrm{N})$ in intersection regions.

Definition 3: $\mathrm{j} \in \mathrm{J}=\{0,1,2,3 \ldots \mathrm{M}\}$ represents $\mathrm{M}$ kinds of faults, like fault 1 , fault $2 \ldots$ fault $\mathrm{M}$.

Definition $4: \mathrm{k} \in \mathrm{K}=\{0,1,2,3\}, 0$ represents normal connection state, 1 represents route discovery state, 2 represents route reconstruction wait state. 3 represent no available routing nodes.

Definition 5: $(\mathrm{N}, 0,0)$ represents normal connection status. Define that there are $\mathrm{N}$ nodes in intersection regions, and $\mathrm{N}-1$ backup nodes.

Definition 6: $(\mathrm{N}-1,0,0)$ represents that the system works regularly, and one of the routing exchange nodes becomes invalid.

Definition 7: $(\mathrm{N}-1,1,1)$ represents system disconnection. The system is in a state of route discovery. The numbers of available nodes are $\mathrm{N}-1$. There is the first type of faults which causes one of the nodes invalid.

Definition 8: $(1,0,0)$ represents that there is one node available, and no backup nodes.

Definition 9: $(0,0,3)$ represents no nodes available and no routing nodes. The possible reasons might be several faults or nodes being invalid (nodes not in the intersection regions), or route discovery. 
Definition 10: $(1,0,2)$ represents one routing node available. The system is in a state of route reconstruction. And none of nodes have been found so far.

Definition 11: $(0, \mathrm{j}, 3)$ represents no available routing nodes. The possible reasons might be several faults or nodes being invalid (nodes not in the intersection regions), or in a state of route discovery.

According above definitions, it can build a continuous-time Markov course model (CTMC) of Ad hoc network (as shown in Figure 2). Define nodes' failure rate (or the rate of departing from the intersection regions) as $\lambda$, the average repair rate of all kinds of faults (or the rate of returning to the intersection regions) as $\mu$, the possibility of all kinds of faults as $\mathrm{p} 1, \mathrm{p} 2 \ldots \mathrm{pm}$, average transfer delay of all kinds of faults as $1 / \delta 1,1 / \delta 2, \ldots 1 / \delta \mathrm{m}$ and average reconnection delay as $1 / \delta \mathrm{w}$.

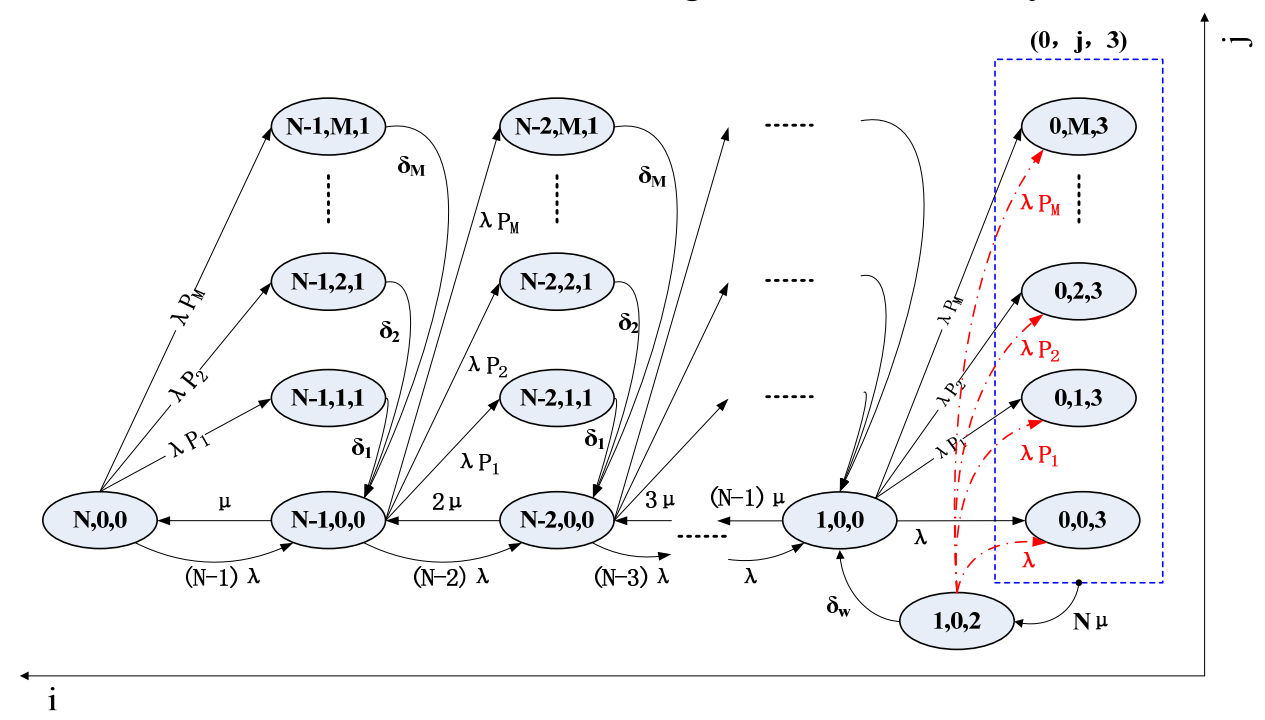

Figure 2 Continuous-time Markov course model (CTMC) of Ad hoc network

The model works as follows:

When the system is in a state of normal connection $(\mathrm{N}, 0,0), \mathrm{N}$ nodes are in the intersection regions and work regularly. When one of nodes tries to forward data, any one of nodes among N-1 nodes left might become invalid in a rate of $\lambda$. When it turns to state $(\mathrm{N}-1,0,0)$, the system is in a state of normal connection, and backup routing nodes decrease to $\mathrm{N}-2$.

If the invalid node tries to repair in the average repair rate of $\mu$, the state would change from ( $\mathrm{N}-1$, $0,0)$ to $(\mathrm{N}, 0,0)$. If fault $\# 1$ occurs in a possibility of $\mathrm{p} 1$, the state will change from $(\mathrm{N}-1,0,0)$ to $(\mathrm{N}-1$, $1,1)$. It represents disconnection, and the system is in a state of route discovery. Besides, the number of available routing nodes is $\mathrm{N}-1$.

If fault $\# 2$ occurs in a possibility of $\mathrm{p} 2$, the state will change from $(\mathrm{N}-1,0,0)$ to $(\mathrm{N}-1,2,1)$. It represents disconnection, and the system is in a state of route discovery. Besides, the number of available routing nodes is $\mathrm{N}-1$. And so on. As for fault \#1, the state will transfer to $(\mathrm{N}-1,0,0)$ after average delay of $1 / \delta 1$. As for fault $\# 2$, the state will transfer to $(\mathrm{N}-1,0,0)$ after average delay of $1 / \delta 2$.

Assumed that the initial state is $(1,0,0)$, if the last node fails or departs from intersection regions, there will be no nodes available for route reconstruction. So the state couldn't transfer to $(0, j, 3)$. Under the circumstance, $\mathrm{N}$ backup nodes are able to repair in an average rate of $\mu$. Then the state will shift from $(0, \mathrm{j}, 3)$ to $(1,0,2)$, waiting for reconnection. If there are nodes available in intersection regions, the system will be reconnected and the state would transfer to $(1,0,0)$. But if node fails or departs from intersection regions again, the state will transfer to $(0, \mathrm{j}, 3)$.

Definition 12: The state shifts from $(1,0,2)$ to $(1,0,0)$ after a transfer delay $\delta w$. By that time, the route has been restored. $\delta \mathrm{w}$ is called average delay of reconnection. $\delta \mathrm{w} \cong \min \{\delta 1, \delta 2 \ldots \delta \mathrm{m}\}$

$\pi_{i, j, k}$ are corresponding probabilities of steady-state availability. Assumed $\rho=\mu / \lambda$, $\theta=\delta_{W} / \lambda+\delta_{W}$, after calculation, it can draw a conclusion:

$$
\pi_{\mathbf{0}, \mathrm{j}, 3}=\left[1+\frac{N \mu}{\lambda+\delta_{w}}+\sum_{i=1}^{N}\left(\begin{array}{c}
N \\
i
\end{array}\right) \rho^{i} \theta+\sum_{j=1}^{M} \sum_{i=1}^{N-1}\left(\begin{array}{c}
N \\
i+1
\end{array}\right)\left(\frac{P_{j}}{\delta_{j}}\right) \rho^{i} \theta\right]^{-1}
$$




$$
\pi_{i, \mathbf{0 , 0}}=\frac{N !}{i !(N-i) !} \rho^{i} \theta \pi_{0, \mathrm{j}, 3}
$$

By CTMC model, it can draw conclusions as followed:

Theorem 1: When the state is between $(\mathrm{N}, 0,0)$ to $(1,0,0)$, the system is in a state of normal connection. Moreover, all of these states are stable. So the system's end-to-end steady-state availability could be represented by the following formula:

$$
\mathrm{A}_{s}=\sum_{i=1}^{N} \pi_{i, 0,0}
$$

Theorem 2: When the state is between $(\mathrm{N}, 0,0)$ to $(1,0,0)$, the system is in a state of normal connection. Due to the node redundancy, once the state transfer from $(1,0,0)$ to $(0,0,0)$, it shows that the connection is in abnormal state. Thus, the connecting probability can be represented by the following formula:

$$
C=1-\pi_{0,0,0}
$$

\section{Establishment of node transient analysis model.}

Influence on network caused by exceptions needs to be taken into consideration, if we try to conduct a comprehensive assessment of Ad hoc network. Too much instantaneous flow will lead to overload when exceptions or faults occur, which might cause connection failure. At the same time, the model for Ad hoc network on steady-state availability couldn't make sense of above situation. The only way to solve it is to analyze the instantaneous data processing based on steady-state model.

As for communications among nodes in Ad hoc network, it can model and analyze instantaneous data processing using $\mathrm{M} / \mathrm{M} / 1 / \mathrm{K}$ queuing theory at the premise of the steady state and availability. Assumed that data processing at nodes in Ad hoc network as shown in Figure 3, $\lambda p$ is packet arrival rate, $\mu$ p is packet transfer rate and $\mathrm{K}$ is queue length. $\mathrm{i} \in \mathrm{I}=\{0,1,2, \ldots, \mathrm{K}\}$ represents the state of $\mathrm{M} / \mathrm{M} / 1 / \mathrm{K}$, and it shows the current length of queue.

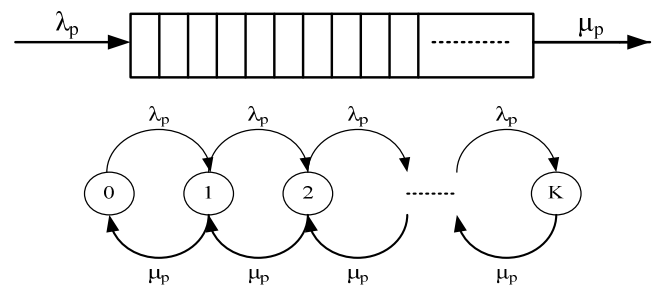

Figure 3 data processing based on $\mathrm{M} / \mathrm{M} / 1 / \mathrm{K}$ queuing theory

Assumed that $P_{m}(i, t)$ represents the probability at the time of $\mathrm{t}$, when CTMC is in the state $\mathrm{i}$. Here are several difference equations:

$$
\begin{aligned}
\frac{d P_{m}(0, t)}{d t}= & -\lambda_{p} P_{m}(0, t)+\mu_{p} P_{m}(1, t) \quad i=0 \\
\frac{d P_{m}(i, t)}{d t}=- & -\left(\lambda_{p}+\mu_{p}\right) P_{m}(i, t)+\lambda_{p} P_{m}(i-1, t)+ \\
& \mu_{p} P_{m}(i+1, t) \quad K>i \geq 1 \\
\frac{d P_{m}(K, t)}{d t}= & -\mu_{p} P_{m}(K, t)+\lambda_{p} P_{m}(K-1, t) \quad i=K
\end{aligned}
$$

When $\frac{d P_{m}(i, t)}{d t}=0$, based on above difference equations, it can obtain steady-state probability $P_{m}(i)$ and following formulas:

$$
\begin{gathered}
P_{m}(i)=\rho_{p}^{i} P_{m}(0) \quad 0<i \leq K \\
P_{m}(0)=1 / \sum_{i=0}^{K} \rho_{p}^{i}=\left\{\begin{array}{ll}
\frac{1-\rho_{p}}{1-\rho_{p}^{K+1}} & \rho_{p} \neq 1 \\
\frac{1}{K+1} & \rho_{p}=1
\end{array}, \quad\left(\rho_{p}=\lambda_{p} / \mu_{p}\right)\right.
\end{gathered}
$$

When connection fails, $\mu_{p}=0$, and queuing model (as shown in the Figure 3) evolves into a finite state birth process. Assumed that $P(i \mid k, t)$ represents the probability of i packets in the queue at the 
time of $\mathrm{t}$ and $\mathrm{k}(\mathrm{k}<=\mathrm{K})$ packets in buffer queue during connection failure. So it can obtain Kolmogorov differential equation in birth processes as following formulas:

$$
\begin{aligned}
& P_{n}(i \mid k, t)=0 \quad 0 \leq i<k \leq K \\
& \frac{d P_{n}(i \mid k, t)}{d t}=\lambda_{p} P_{n}(i-1 \mid k, t)-\lambda_{p} P_{n}(i \mid k, t) \\
& K>i \geq k \geq 0 \\
& \frac{d P_{n}(K \mid k, t)}{d t}=\lambda_{p} P_{n}(K-1 \mid k, t) \quad i=K
\end{aligned}
$$

By solving instantaneous CTMC process [10], it can obtain:

$$
P_{n}(i \mid k, t)=\left\{\begin{array}{cc}
0 & i<k \leq K \\
\frac{(\lambda t)^{i-k}}{(i-k) !} e^{-\lambda_{p} t} & K>i \geq k \\
1-\sum_{j=0}^{i-K} \frac{(\lambda t)^{j}}{j !} e^{-\lambda_{p} t} & i=K
\end{array}\right.
$$

There have been $\mathrm{k}$ packets at the time of $\mathrm{t}$. And the probability of full buffer is:

$$
P_{n}(K \mid k, t)=1-\sum_{j=0}^{K-k} \frac{(\lambda t)^{j}}{j !} e^{-\lambda_{p} t}
$$

Assumed that $t_{x}$ represents continuous failure events and there has been $\mathrm{k}$ packets before the buffer becomes invalid. So before buffer's invalidity, packet losses would be:

$$
l_{r}\left(k, t_{x}\right)=\int_{0}^{t_{x}} \lambda_{p} P_{n}(K \mid k, t) d t
$$

If the queue is full at the time of $t$, the probability of full buffer is $P_{n}(K \mid k, t)$. In the duration of end-to-end connection failure, there would be no packets. But after that, the packets would be dropped. So the number of packet losses is:

$$
L_{r}\left(k, t_{x}\right)=\int_{0}^{t_{x}} \lambda_{p} P_{n}(K \mid k, t) d t-\lambda_{p} P_{m}(K)
$$

To evaluate the influence instantaneous overload has on the network, document [11] introduces two indexes: Expected Excess Loss in Overload (EELO) and Expected Excess Delay in Overload (EEDO). In consideration of connection failure at any time and packet number $\mathrm{k}$ being a random variable, supposed that the state has been stable before connection failure, the average EELO would be:

$$
\operatorname{EELO}\left(t_{x}\right)=\sum_{k=0}^{K} l_{r}(k, t) P_{m}(k)-\lambda_{p} P_{m}(K) t_{x}
$$

Transfer delay $t_{x}$ is a random variable too. Supposed that duration of continuous connection failure $^{t_{x}}$,s probability distribution function is $P_{s}\left(t_{x}\right)$, so EELO can be:

$$
\begin{aligned}
\left.E E L O\right|_{t_{x}, P_{s}\left(t_{x}\right)}= & \sum_{k=0}^{K} \int_{0}^{\infty} l_{r}\left(k, t_{x}\right) P_{m}(k) P_{s}^{\prime}\left(t_{x}\right) d t_{x}- \\
& \int_{0}^{\infty} \lambda_{p} P_{m}(K) t_{x} P_{s}^{\prime}\left(t_{x}\right) d t_{x}
\end{aligned}
$$

As for real-time application of network, delay is a very important parameter. Supposed that duration of continuous connection failure $t_{x}$, s probability distribution function is $P_{s}\left(t_{x}\right)$, so EEDO can be:

$$
\left.E E D O\right|_{t_{x} \sim P_{s}\left(t_{x}\right)}=\int_{0}^{\infty} t_{x} P_{s}^{\prime}\left(t_{x}\right) d t_{x}
$$

\section{Establishment of composite survivability assessment model.}

Survivability in Ad hoc network includes two parts: steady-state availability and instantaneous influence in exception conditions. The above two can be combined into one model called composite survivability model. In consideration of mobility of nodes, nodes and link faults, it could build a 
continuous Markov process model for Ad hoc network. With the model, it could assess fault frequency and steady-state availability of the system; moreover, it could assess the possible duration of exceptions and influence exceptions have on the network by instantaneous analysis of system with queuing model. In a word, based on comprehensive analysis of above model, it could establish composite survivability analysis model for Ad hoc network by extending indexes EELO and EEDO.

Definition 13: Loss Due to Failures (LDF) could be obtained by the equations 1 to 5:

$$
\begin{aligned}
& L D F=\sum_{j=1}^{N}\left(\lambda \pi _ { j , 0 , 0 } \left(\left.p_{1} E E L O\right|_{t_{x} \sim E X P\left(1 / \delta_{1}\right)}+\left.p_{2} E E L O\right|_{t_{x} \sim E X P\left(1 / \delta_{2}\right)}+\right.\right. \\
& \left.\left.\left.p_{3} E E L O\right|_{t_{x} \sim \operatorname{EXP}\left(1 / \delta_{3}\right)}+\cdots+\left.p_{m} E E L O\right|_{t_{t_{x}} \in \operatorname{EXP}\left(1 / \delta_{m}\right)}\right)\right)+\left.\lambda \pi_{1,0,0} E E L O\right|_{t_{x} \sim \operatorname{EXP}(1 / N \mu)}
\end{aligned}
$$

Definition 14: Delay Due to Failures (DDF) could be obtained by the following equations:

$$
\begin{aligned}
D D F & =\sum_{j=1}^{N}\left(\lambda \pi _ { j , 0 , 0 } \left(\left.p_{1} E E D O\right|_{t_{x} \sim \operatorname{EXP}\left(1 / \delta_{1}\right)}+\left.p_{2} E E D O\right|_{t_{x} \sim \operatorname{EXP}\left(1 / \delta_{2}\right)}+\right.\right. \\
\left.\left.\left.p_{3} E E D O\right|_{t_{x} \sim \operatorname{EXP}\left(1 / \delta_{3}\right)}+\cdots+\left.p_{m} E E D O\right|_{t_{x} \sim \operatorname{EXP}\left(1 / \delta_{m}\right)}\right)\right)+\left.\lambda \pi_{1,0,0} E E D O\right|_{t_{x} \sim E X P(1 / N \mu)} & =\sum_{j=1}^{N}\left(\lambda \pi_{j, 0,0}\left(\frac{p_{1}}{\delta_{1}}+\frac{p_{2}}{\delta_{2}}+\cdots+\frac{p_{m}}{\delta_{m}}\right)\right)+\lambda \pi_{j, 0,0} \frac{1}{N \mu} \\
= & \sum_{j=1}^{N}\left(\lambda \pi_{j, 0,0} \sum_{i=1}^{m} \frac{p_{m}}{\delta_{m}}\right)+\lambda \pi_{j, 0,0} \frac{1}{N \mu}
\end{aligned}
$$

As, LDF and DDF are used to assess quantitatively influence exceptions have on survivability of Ad hoc network. The parameter As represents the probability of link availability in steady state. Under invalid conditions, instantaneous overload could be represented by parameters LDF and DDF.

The above model is built at the premise of double hop transmission. As for multiple hop transmission, it can analyze those parameters and build survivability model based on reliability theory. The following figure 4 shows data transmission in the situation of multiple hop transmission.

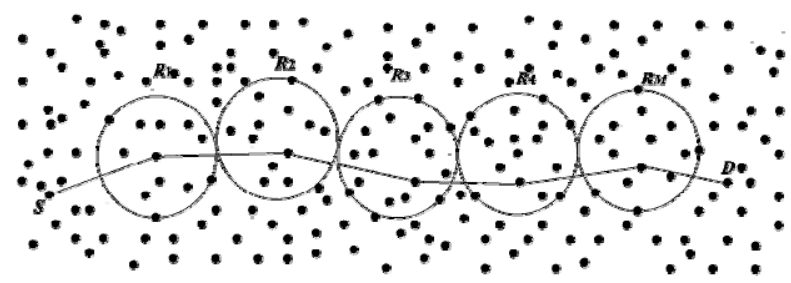

Figure 4 data transmission in the situation of multiple hop transmission in Ad hoc network

Definition 15: Multiple hop transmission is like multiple double hop transmission connecting in series with each others. The steady state availability could be obtained by formula for computing reliability of series systems. In the situation of multiple hop transmission, system availability could be represented by $A_{L}$ :

$$
A_{L}=\prod_{i=1}^{L} A s
$$

Definition 16: In the process of multiple hop transmission, Delay Due to Failures (DDF) and Loss Due to Failures (LDF) are a cumulative process when in series systems. So the $D D F_{L}$ and $L D F_{L}$ are respectively:

$$
D D F_{L}=\sum_{i=1}^{L} D D F \quad ; \quad L D F_{L}=\sum_{i=1}^{L} L D F
$$

Above all, it can draw a conclusion that indexes for connecting survivability assessment are $A_{L}$, $D D F_{L}$ and $L D F_{L}$.

\section{Model verification}

Through simulation analysis made by MATLAB, it can analyze variation rules of steady state availability and instantaneous packet losses and delay in Ad hoc network. Setting parameters: 
Defined that hops in data transmission process is $\mathrm{L}$, nodes in intersection regions are $\mathrm{N}$, arrival rate of packets $\lambda$ p ranges from 1 to 10 , packet transfer rate $\mu$ p equals to 25 , queue length $K$ equals to 200 . Assumed that there are 5 types of faults in Ad hoc network, and all the faults have the same occurring rates: $\mathrm{p} 1=\mathrm{p} 2=\mathrm{p} 3=\mathrm{p} 4=\mathrm{p} 5=0.2$. Node failure rate $\lambda$ ranges from 0.01 to 0.1 . Average fault repair rate $\mu$ equals to 0.5 . Average fault transfer delays are respectively: $1 / \delta 1=1 / \delta 2=1 / \delta 3=1 / \delta 4=1 / \delta 5=1 / \delta \mathrm{w}=0.01$. Simulation result is shown in Figure 5:

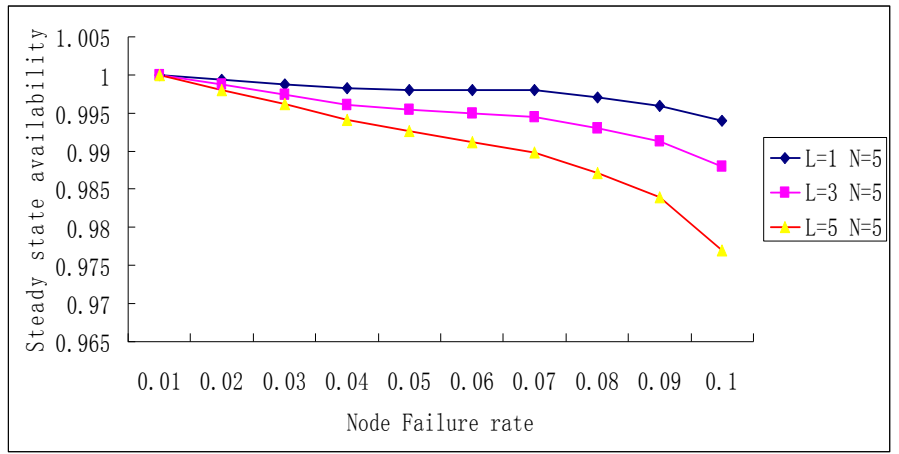

Figure 5 Steady state availability in multiple hop transmission

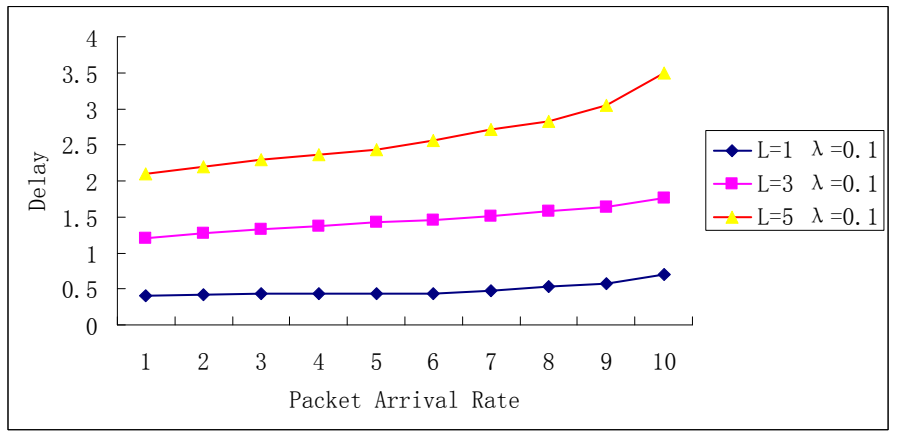

Figure 6 delay in multiple hop transmission

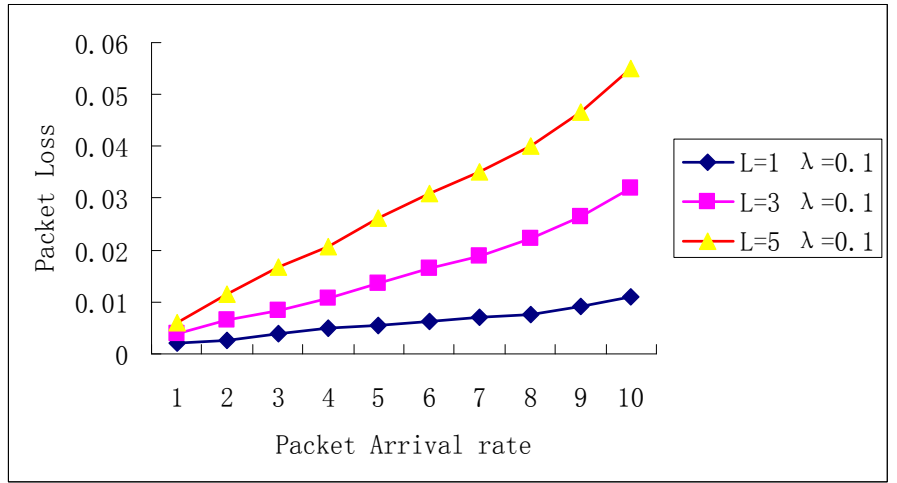

Figure 7 pocket loss rate in multiple hop transmission

Figure 5 shows the relationship between node failure rate $\lambda$ and steady state availability AL. AL decreases as $\lambda$ increases. Meanwhile, when hops in data transmission increase, AL decreases.

Figure 6 shows that while packet arrival rate $\lambda p$ increases, DDFL increases too. The reason why DDFL increases is because that when $\lambda p$ increases, more packets in the queue waiting for being processed. Thus, prolong the time of packet processing.

Figure 7 shows that while packet arrival rate $\lambda p$ increases, LDFL increases too. The reason for it is that with the increasing of packet arrival, packets are dropped due to not timely processing excess packets in queue.

\section{Summary}

As for research on survivability of Ad hoc network, it should assess the connecting reliability firstly. And then make a further survivability analysis of its quality and service. This article introduces a composite quantitative assessment model. And the model makes analysis of steady-state 
availability and link overload in the situation of connection failure. The result of this experiment shows that the model is practical and effective.

\section{References}

[1] DEEP M, DAVID T. Multi-Layered Network Survivability Models, Analysis, Architecture, Framework and Implementation: An Overview [C]. Proceeding of the 2000 DARPA Information Survivability Conference \& Exposition. 2000:173-186.

[2] Zhang Hui Min, Gu Tian Long. Modeling and analyzing techniques for networks survivability [J]. Journal of Guilin University of Electronic Technology, 2006, 26(5): 370-374. (In Chinese)

[3] Chen D Y, Garg S, Trivedi K S. Network survivability performance evaluation: A quantitative approach with applications in wireless Ad Hoc networks [C]. ACM. MSWiM'02, Atlanta, September2002.

[4] John Koroma, Wei Li, Demetrios Kazakos. A generalized model for network Survivability [C]. ACM. TAPIA’03, October, 15-18, 2003.

[5] Peng San Cheng, Jia Wei Jia, Wang Guo Jun. Survivability evaluation in large scale mobile Ad Hoc networks [J]. Journal of Computer Science and Technology, 2009, 24(4):761-774.

[6] Krishna Paul, Romit Roy Choudhuri, Somprakash Bandyopadhyay. Survivability Analysis of Ad Hoc Wireless Network Architecture [C]. Proceedings of the IFIP-TC6/European Commission International Workshop on Mobile and Wireless Communication Networks. London: Springer-Verlag, 2000: 31-46.

[7] Liu Mei Xia, Gu Tian Long, Survivability Analysis of Ad Hoc Network Based on GSPN Model [J]. Journal of Guilin University of Electronic Technology, 2009, 29(2): 82-87.

[8] Ellison R, Linger R. Survivable Network System Analysis: a Case Study [J]. Software, 1999, 16(4):70-77

[9] Knight J, Sullivan K. On the Definition of Survivability [R]. Department of Computer Science, Technical Report CS200233, Virginia: University of Virginia, 2000

[10]K.S.Trivedi. Probability \& Statistics with Reliability, queuing, and Computer Science Applications [M]. John Wiley \& Sons, second edition, 2001.

[11]T1A1.2Working Group on Network Survivability Performance [R]. Technical report on enhanced network survivability performance, February 2001. 